\title{
EDITORIAL
}

\section{Scientific Publications and the Internet}

The Internet is a potent medium for the global distribution and retrieval of information in all areas of life. We already profit from this opportunity and will do so even more when the Internet will be developed further. But there is another side to all this: The fact that unchecked information is available to an almost unlimited extent also carries dangers with it particularly when it is given the semblance of being based on scientific evidence. For the publication of scientific knowledge, an efficient control system has matured and proved successful over many years: peer review. The Internet is not the only medium to dodge this system, but the Internet is certainly the cheapest, fastest, most widespread and therefore the most efficient (and probably dangerous) way to do so. A recent example is Arpad PUSZTAI's claim in the press and on television programmes in Great Britain that genetically modified (GM) potatoes may stunt the growth of rats. His "findings", although not published in a peer-reviewed journal, were welcomed and exploited by lobby groups and triggered widespread concern over the issue of GM food. A year later the paper was published in The Lancet accompanied by a critical commentary (A. Pusztai: Lancet 354 (1999) 1314-1315). One benefit of the publication in this prestigious journal certainly was that PUSZTAI had to retract his original claims because the data simply do not support them (N. Loder: Nature 401 (1999) 731).

A similar case, we believe, is the Internet publication of July 14, 1999, on the website of 'Action on Smoking and Health' (ASH) entitled "Tobacco Additives - Cigarette Engineering and Nicotine Addiction", authored by C. BATES, M. JARVIS and G. CONNOLLY (http://www.ash.org.uk/papers/additives.html). In this report, the authors claim, among other things, that the cigarette industry uses pharmacologically active additives in order to influence the smoking behaviour. The scientific evidence for their claim is mainly based on notes and memos from scientists of the tobacco industry which are also available on the Internet. As in the example discussed above, the ASH paper achieved a widespread coverage in the media, impressing not only the public but also government bodies, thus certainly serving its intended purpose. It is our view that legislation based on (or at least influenced by) dubious scientific evidence is harmful not only for the industry but even more for those who are supposed to be protected, the consumers. We, therefore, believe that the invited Commentary by L. MÜLLER and W. RÖPER (see page 51) on only a few aspects of the ASH paper on additives is of interest for our readers. The Mini-Review by DIXON et al. (page 103) in appropriate scientific depth tackles the problem of nicotine transfer from tobacco to the smoker and the role of ammonia compounds used in the production of cigarettes. This Mini-Review is the first of a series of peer-reviewed, 'state-of-the-art' overviews on issues of current interest in tobacco research. The Mini-Review will present its subject as a complete scientific manuscript accompanied by headlines allowing a quick overview of the main issues. Furthermore, we as editors of Beiträge zur Tabakforschung International take the liberty but also the responsibility to invite experts to write Commentaries on selected topics. Commentaries represent scientifically based views of the authors and are intended to encourage further scientific discussion.

ALLEN et al. (Nature: 402 (1999) 722) reported on the overall reliability of scientific information on websites and found that a high percentage was 'misleading', 'inaccurate' and 'unreferenced'. Nonetheless, the authors recognize the substantial advantages of the Internet as a resource for scientific information provided that peer review is maintained as the guiding principle for evaluating science.

We deeply regret that the circulation of our journal is not sufficient to correct this kind of misinformation. In order to at least partly remedy this disadvantage, but also for other reasons, we as editors of Beiträge zur Tabakforschung International are considering a full-text Internet version (besides the printed version) of this journal to be implemented in the year 2001. In consideration of our comments above, we, however, want to assure our readers that the Beiträge will retain its peer-review system. 